\title{
Full Quantum Analysis of Complete Population Transfer Using Frequency Boost
}

\author{
Fatemeh Ahmadinouri $^{\mathrm{a}}$, Mehdi Hosseini ${ }^{\mathrm{a}^{*}}$, and Farrokh Sarreshtedari ${ }^{\mathrm{b}}$

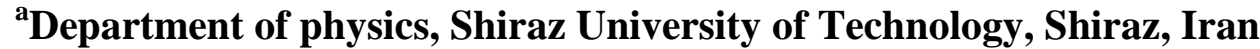 \\ ${ }^{b}$ Department of Physics, University of Tehran, Tehran, Iran \\ *Corresponding Author's Email: hosseini@sutech.ac.ir
}

\begin{abstract}
Regular Paper: Received: Aug. 24, 2020, Revised: Apr. 26, 2020, Accepted: May. 3, 2020, Available Online: May. 4, 2020, DOI: 10.29252/ijop.14.1.91
\end{abstract}

\begin{abstract}
In this paper, we have proposed and demonstrated a new method of atomic population transfer. The transition dynamic of a two-level system is studied in a full quantum description of the Jaynes-Cummings model. Solving the timedependent Schrödinger equation, we have investigated the transition probabilities numerically and analytically by using a sudden boost of the laser frequency. The results show that complete population transfer can be achieved by adjusting the time of the frequency boost.
\end{abstract}

KEYWORDS: population transfer, two-level system, Rabi oscillation, Jaynes-Cummings model.

\section{INTRODUCTION}

Population transfer of quantum states plays a fundamentally important role in a variety of fields of physics [1-5]. For this reason, finding an efficient transferring mechanism is an important issue in atomic physics. Frequencychirped laser [1, 6-8], Stimulated Raman Adiabatic Passage (STIRAP) [9,10], and Landau-Zener technique $[1,12,13]$ are prominent approaches in stable population transfer which have many experimental and theoretical applications in quantum optics.

One of the fundamental schemes for full quantum modeling of the system dynamics is the Jaynes-Cummings (JC) model [13-15]. JC model explains analytically the interaction of a quantized electromagnetic field with a two- level system. The early history of this model returns to magnetic resonance [16-17] and it was presented in 1963 by Jaynes and Cummings [18]. The JC model is experimentally realized with the high-Q superconducting cavities and a Rydberg atom [19]. This scheme describes several interesting phenomena such as collapse and revivals [20], atom-field entanglement [19, 21], squeezing [22-24], and Rabi Oscillation (RO) [15].

Rabi Oscillation was first introduced in 1937 and explained by the interaction of the oscillating magnetic field and the magnetic moments [25]. The Rabi oscillations mainly describe the interaction of the two-level atom with a radiation field [26]. Such behavior is observed in the different fields such as quantum dots [27, 28], trapped ions [29, 30], superconducting quantum devices [31], semiconductors [32], surface plasmons [33], Bose-Einstein condensates [34], diamond nitrogen-vacancy centers [35] and Josephson junction qubits [36].

Here, we have numerically and analytically studied the population transfer in a two-level system using the Jaynes-Cummings model. In this method, the variation of Rabi Oscillations and transition probabilities are investigated using sudden frequency change and it is shown that by tuning the time of frequency boost, the complete population transfer can be obtained. 


\section{TheORETICAL MODEL}

In this work, the transition probabilities have been studied using a full quantum model of the Jaynes-Cummings (JC). When an external electric field interacts with a two-level system, the JC Hamiltonian (Eq. 1) includes three terms for the atom, field, and interaction between them respectively as following [37]:

$$
\begin{aligned}
& H=-\frac{1}{2} \hbar \omega_{0} \sigma_{z}+\hbar \omega_{L}\left(a^{\dagger} a+\frac{1}{2}\right) \\
& +\hbar \omega_{1}\left(a \sigma_{+}+a^{\dagger} \sigma_{-}\right)
\end{aligned}
$$

where $\hbar$ is Planck constant, $\omega_{0}$ is the atomic transition frequency, $\omega_{L}$ is the laser frequency. As well as $a, a$ are annihilation and creation operators respectively, and $\sigma_{\text {-, }} \sigma_{+}, \sigma_{z}$ are Pauli spin matrices. In this definition, $\omega_{1}$ describes the coupling strength between atom and field which is determined by the atomic transition dipole moment and the laser field and is proportional to Rabi frequency [37]:

$$
\Omega=\sqrt{\left(\omega_{L}-\omega_{0}\right)^{2}+\left(2 \omega_{1}\right)^{2}}
$$

By acting the JC Hamiltonian (Eq. 1) on eigenstates $|g, n\rangle$ (ground state, $n$ photons) and $|e, n-1\rangle$ (excited state, $n$ photons) (Eq. 3), the final Hamiltonian (Eq. 4) can be obtained [37].

$$
\begin{aligned}
& H_{\text {atom }}|g ; n\rangle=-\frac{1}{2} \hbar \omega_{0}|g ; n\rangle \\
& H_{\text {field }}|g ; n\rangle=\hbar \omega_{L}\left(n+\frac{1}{2}\right)|g ; n\rangle \\
& H_{\mathrm{int}}|g ; n\rangle=\hbar \omega_{1} \sqrt{n}|e ; n-1\rangle \\
& H_{\text {atom }}|e ; n-1\rangle=\frac{1}{2} \hbar \omega_{0}|e ; n-1\rangle \\
& H_{\text {field }}|e ; n-1\rangle=\hbar \omega_{L}\left(n-\frac{1}{2}\right)|e ; n-1\rangle \\
& H_{\mathrm{int}}|e ; n-1\rangle=\hbar \omega_{1} \sqrt{n}|g ; n\rangle
\end{aligned}
$$

Finally, by using this Hamiltonian (Eq. 4) and by numerically solving the time-dependent Schrödinger equation the transition probabilities are obtained using Runge-Kutta method. In this calculation, $n$ is equal to one.

$$
H=\hbar\left(\begin{array}{cc}
\left(n+\frac{1}{2}\right) \omega_{L}-\frac{\omega_{0}}{2} & \omega_{1} \sqrt{n} \\
\omega_{1} \sqrt{n} & \left(n-\frac{1}{2}\right) \omega_{L}+\frac{\omega_{0}}{2}
\end{array}\right)
$$

It is worth mentioning that for simplicity, all the parameters are dimensionless by the relation (5) in our calculations.

$$
\tilde{\omega}_{0}=1, \tilde{t}=t \omega_{0}, \tilde{\omega}_{1}=\frac{\omega_{1}}{\omega_{0}}, \tilde{\omega}_{L}=\frac{\omega_{L}}{\omega_{0}}
$$

\section{RESULTS AND DISCUSSION}

As mentioned before, when a two-level atom interacts with a resonant external field, the system population oscillates between the ground and excited states. Here, the system population transfer and variation of Rabioscillations have been investigated by a sudden change in laser frequency towards achieving the stable and complete population transfer.

In section $A$, the effect of a sudden increase in the laser frequency on the transition probabilities has been investigated. In section $\mathrm{B}$, the transition probabilities using two frequency boosts have been studied. Moreover, it is shown that the analytical solution of transition probabilities is in agreement with the numerical solution in section $\mathrm{C}$.

\section{A. Sudden increase in the laser frequency}

In Fig. 1, the transition probabilities versus time are depicted for $\tilde{\omega} 1=0.1$. The initial and final normalized laser frequency is considered $\tilde{\omega}_{a}$ and $\tilde{\omega}_{b}$, respectively. In order to investigate the effect of the sudden increase of the laser frequency, the time of the frequency change is set when the probability of the initial ROs is minimum and maximum in the left and right column in Fig. 1, respectively. Also in the middle column, this frequency increase is tuned when the probability of the initial ROs is in half of the maximum. This figure reveals 
that by the sudden increase in the laser frequency i) the amplitude of the probability oscillation is intensively decreased and whereby the final probability stability is enhanced. ii) The probability of system population transfer remains constant at the same probability of the frequency boost time. Therefore, it is possible to achieve the desired probability by adjusting the time of the sudden increase of the laser frequency. Indeed, the boosting time plays a key role in the final probability. So, in order to achieve the maximum possible probability, the time of the frequency boost is set to the time that the probability of the initial ROs is maximum (right column). Accordingly, the complete transition (Fig. 1 (f)) is observed when the initial ROs oscillate between zero and one (middle row). It means that the initial frequency is tuned to the atomic transition frequency $\left(\tilde{\omega}_{a}=1\right)$. This figure also shows that by decreasing (upper row) or increasing (lower row) the initial frequency, probabilities are reduced.

In [19] it has been shown that by applying the chirped laser source in a limited time interval, the population of the states can be tuned in which the results are similar to the method of the frequency boost that is proposed here.
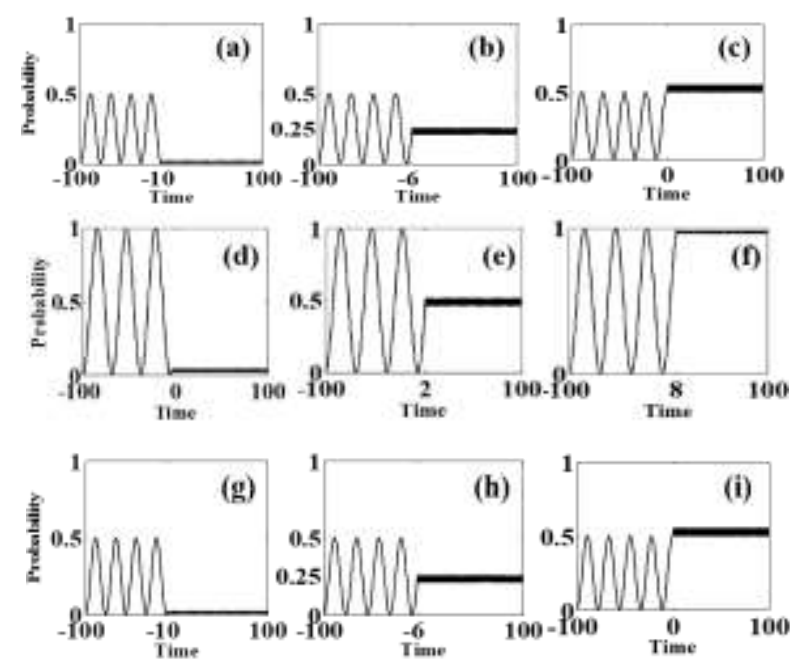

Fig. 1. Transition probability versus time $-100<$ $\tilde{t}<100, \tilde{\omega}_{l}=0.1$ (a), (b), (c): $\tilde{\omega}_{a}=0.8, \tilde{\omega}_{b}=5$ (d), (e), (f): $\tilde{\omega}_{a}=1, \tilde{\omega}_{b}=5(\mathrm{~g}),(\mathrm{h}),(\mathrm{i}): \tilde{\omega}_{a}=1.2, \tilde{\omega}_{b}=5$.

The transition probability versus time for different final laser frequencies is illustrated in
Fig. 2, in which the initial laser frequency is equal to the atomic transition frequency. The time of the frequency boost is adjusted when the probability of the initial ROs is half of the maximum (0.5). This figure shows that the amplitude of the secondary ROs is reduced by increasing the final laser frequency. Therefore, by increasing the frequency differences, stable population transfer with the lowest oscillation can be achieved.

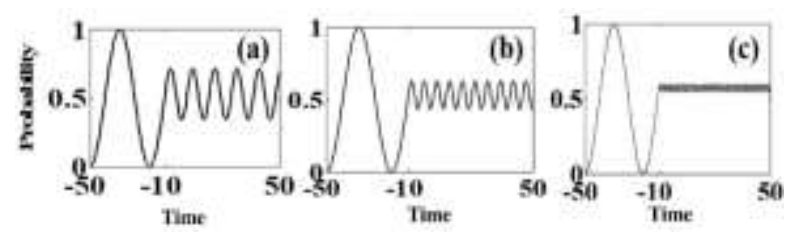

Fig. 2. Transition probability versus time $-50<$ $\tilde{t}<50, \quad \tilde{\omega}_{l}=0.1, \quad \tilde{\omega}_{a}=1$ (a) $\tilde{\omega}_{b}=1.5$ (b) $\tilde{\omega}_{b}=2$ (c) $\tilde{\omega}_{b}=5$.

In Fig. 3 transition probability versus time is depicted for different coupling strengths while $\tilde{\omega}_{a}=1, \quad \tilde{\omega}_{b}=5$. The time of the frequency changing is tuned to the complete transition probability. As the ROs frequency increases by increasing the coupling strength, the frequency should change more abruptly to achieve the complete population transfer. In other words, by decreasing the coupling strength, the population transfer would be insensitive to the small variations of the frequency boost time, and thus the robustness increases.
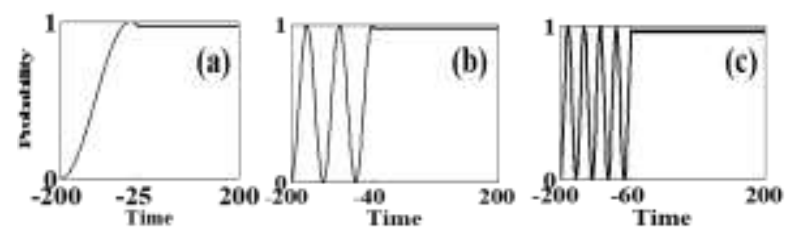

Fig. 3. Transition probability versus time $-200<$ $\tilde{t}<200, \tilde{\omega} a=1, \tilde{\omega}_{b}=5$ (a) $\tilde{\omega}=0.01$ (b) $\tilde{\omega}_{l}=0.05$ (c) $\tilde{\omega}_{l}=0.1$.

It can be inferred that in the sudden frequency boost method, the robust and complete population transfer would be achieved by adjusting the time of the frequency changing, the value of frequency-changing, and coupling strength. 


\section{B. Robust population transfer using two frequency boosts}

So far, the population can be only transferred to the excited state by adjusting system parameters. In this section, the population transfer is investigated in such a way that it ensures that the system has been in the ground state for a long time ago. Then it tries to transfer this population to the excited state. To achieve this goal, the two sudden frequency boosts are proposed. The transition probability versus time for $\tilde{\omega}_{l}=0.1$ is depicted in Fig. 4. The initial, second, and final laser frequencies are considered as $\tilde{\omega}_{a}, \tilde{\omega}_{b}$, and $\tilde{\omega}_{c}$, respectively. As can be seen, $\tilde{\omega}_{b}$ is tuned to the atomic transition frequency. All population is in the ground state if the time of first sudden changing of the laser frequency is set to when the probability of the second ROs is minimum. After that by adjusting the final frequency boost to when the probability of the second ROs is maximum, the stable and complete population transfer occurs.

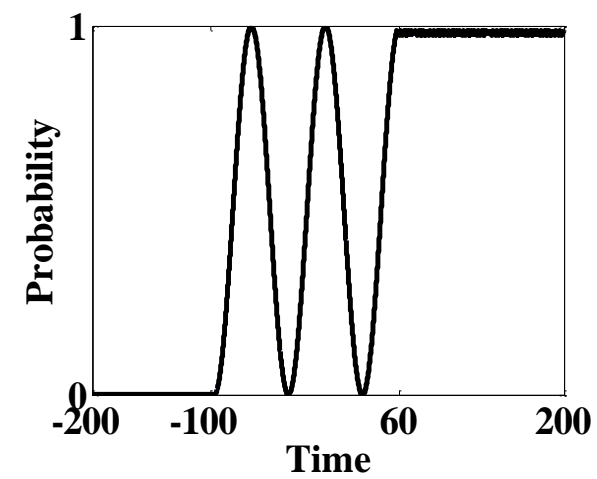

Fig. 4. Transition probability versus time $-200<$ $\tilde{t}<200, \tilde{\omega}_{1}=0.1, \tilde{\omega}_{a}=3, \tilde{\omega}_{b}=1, \tilde{\omega}_{c}=3$.

\section{Analytical solution}

In this section, by analytical solution, the stable and full population transfer to the excited state is investigated. For this aim, the eigenvalue of JC Hamiltonian (Eq. 6) is calculated (Eq. 7).

$$
H=\hbar \omega_{0}\left(\begin{array}{cc}
\frac{1}{2}\left(3 \tilde{\omega}_{L}-1\right) & \tilde{\omega}_{1} \\
\tilde{\omega}_{1} & \frac{1}{2}\left(\tilde{\omega}_{L}+1\right)
\end{array}\right)
$$

$$
\lambda=\hbar \omega_{0}\left(\tilde{\omega}_{L} \mp \frac{\tilde{\Omega}}{2}\right)
$$

Then by solving the time-dependent Schrödinger equation, the wave function is achieved:

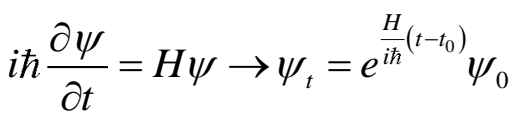

where $\Psi_{0}$ is the wave function in the initial condition, $t_{0}$ is the initial time, $\Psi_{t}$ and $t$ are the final wave function and the final time, respectively.

$$
\begin{aligned}
& \psi_{t}=\psi_{0}\left(R^{-1} e^{\frac{H_{d}\left(t-t_{0}\right)}{i \hbar}} R\right) \\
& H\left(\begin{array}{l}
\psi_{1}(t) \\
\psi_{2}(t)
\end{array}\right)=\lambda\left(\begin{array}{l}
\psi_{1}(t) \\
\psi_{2}(t)
\end{array}\right) \\
& R=\left(\begin{array}{ll}
2 \tilde{\omega}_{1} & -\left(\tilde{\omega}_{L}+\tilde{\Omega}-1\right) \\
-2 \tilde{\omega}_{1} & \left(\tilde{\omega}_{L}-\tilde{\Omega}-1\right)
\end{array}\right)
\end{aligned}
$$

To find the final wave function, we rewrite the Hamiltonian in the diagonal form (Eq. 9). The $d$ index expresses that the Hamiltonian is diagonal. In this solution, $R$ is the operator transformation (Eq. 11) which is obtained by Eq. 10. In this relation, $\Psi_{1}(t)$ and $\Psi_{2}(t)$ are the wave functions in the ground and excited state, respectively.

$$
\begin{aligned}
& \text { a) } \tilde{t}<\tilde{t}_{b s t}:\left(\begin{array}{l}
\psi_{1 a}(\tilde{t}) \\
\psi_{2 a}(\tilde{t})
\end{array}\right)=\frac{1}{\left|-4 \tilde{\omega_{1}} \tilde{\Omega}\right|}\left(\begin{array}{cc}
C_{\tilde{\omega}_{a}}(\tilde{t}) & D_{\tilde{\omega}_{a}}(\tilde{t}) \\
E_{\bar{\omega}_{a}}(\tilde{t}) & F_{\tilde{\omega}_{a}}(\tilde{t})
\end{array}\right)\left(\begin{array}{l}
1 \\
0
\end{array}\right)= \\
& \frac{1}{-4 \tilde{\omega}_{1} \tilde{\Omega} \mid}\left(\begin{array}{c}
C_{\bar{\omega}_{a}}(\tilde{t}) \\
E_{\bar{\omega}_{a}}(\tilde{t})
\end{array}\right)
\end{aligned}
$$

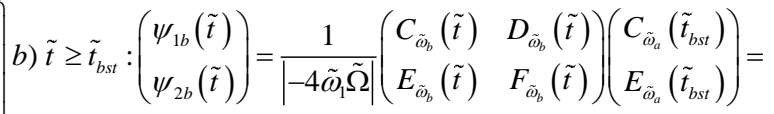

$$
\begin{aligned}
& \frac{1}{-4 \tilde{\omega}_{1} \tilde{\Omega}}\left(\begin{array}{l}
C_{\bar{\omega}_{a}}\left(\tilde{t}_{b s t}\right) C_{\bar{\omega}_{b}}(\tilde{t})+E_{\bar{\omega}_{a}}\left(\tilde{t}_{b s t}\right) D_{\bar{\omega}_{b}}(\tilde{t}) \\
C_{\bar{\omega}_{a}}\left(\tilde{t}_{b s t}\right) E_{\bar{\omega}_{b}}(\tilde{t})+E_{\bar{\omega}_{a}}\left(\tilde{t}_{b s t}\right) F_{\bar{\omega}_{b}}(\tilde{t})
\end{array}\right)
\end{aligned}
$$




$$
\left\{\begin{aligned}
C_{\tilde{\omega}_{L}}(\tilde{t})= & 2 \tilde{\omega}_{1}\left(\tilde{\omega}_{L}-\tilde{\Omega}-1\right) e^{-i\left(\tilde{\omega}_{L}-\frac{\tilde{\Omega}}{2}\right)\left(\tilde{t}-\tilde{t}_{0}\right)}- \\
& 2 \tilde{\omega}_{1}\left(\tilde{\omega}_{L}+\tilde{\Omega}-1\right) e^{-i\left(\tilde{\omega}_{L}+\frac{\tilde{\Omega}}{2}\right)\left(\tilde{t}-\tilde{t}_{0}\right)} \\
D_{\tilde{\omega}_{L}}(\tilde{t})= & \left(\tilde{\omega}_{L}-\tilde{\Omega}-1\right)\left(\tilde{\omega}_{L}+\tilde{\Omega}-1\right) e^{-i\left(\tilde{\omega}_{L}+\frac{\tilde{\Omega}}{2}\right)\left(\tilde{t}-\tilde{t}_{0}\right)}- \\
& \left(\tilde{\omega}_{L}-\tilde{\Omega}-1\right)\left(\tilde{\omega}_{L}+\tilde{\Omega}-1\right) e^{-i\left(\tilde{\omega}_{L}-\frac{\tilde{\Omega}}{2}\right)\left(\tilde{t}-\tilde{t}_{0}\right)} \\
E_{\tilde{\omega}_{L}}(\tilde{t})= & 4 \tilde{\omega}_{1}^{2}\left(e^{-i\left(\tilde{\omega}_{L}-\frac{\tilde{\Omega}}{2}\right)\left(\tilde{t} \tilde{t}_{0}\right)}-e^{-i\left(\tilde{\omega}_{L}+\frac{\tilde{\Omega}}{2}\right)\left(\tilde{t}-\tilde{t}_{0}\right)}\right) \\
F_{\tilde{\omega}_{L}}(\tilde{t})= & 2 \tilde{\omega}_{1}\left(\hat{\omega}_{L}-\tilde{\Omega}-1\right) e^{-i\left(\tilde{\omega}_{L}+\frac{\tilde{\Omega}}{2}\right)\left(\tilde{t}-\tilde{t}_{0}\right)}- \\
& 2 \tilde{\omega}_{1}\left(\tilde{\omega}_{L}+\tilde{\Omega}-1\right) e^{-i\left(\tilde{\omega}_{L}-\frac{\tilde{\Omega}}{2}\right)\left(\tilde{t}-\tilde{t}_{0}\right)}
\end{aligned}\right.
$$

Finally, by solving the Schrödinger equation (Eq. 9), the wave function (Eq. 12) and so the final transition probability can be achieved. In this calculation, $\tilde{t}_{b s t}$ is the time of the frequency boost. In relation (12), the wave function is obtained before (a) and after (b) of the frequency variation. The parameters of this relation are shown in Eq. 13. In this work, it is assumed that the system has been in the ground state for the initial time.
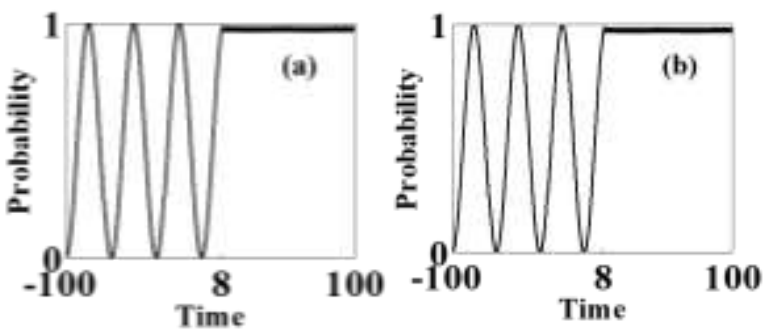

Fig. 5. Transition probability versus time $-100<$ $\tilde{t}<100, \quad \tilde{\omega}_{1}=0.1, \quad \tilde{\omega}_{a}=1, \quad \tilde{\omega}_{b}=5 \quad$ (a) numerical solution (b) analytical Solution.

\section{IV.CONCLUSION}

In this work, the population transfer in a twolevel system has been numerically and analytically studied for a sudden boost of the laser frequency. The population transfer is investigated by numerically solving the Jaynes-Cummings Hamiltonian. This method reveals that by a sudden increase in the laser frequency, the amplitude of the probability oscillation is intensively decreased. In addition, the system probability remains constant at the same probability of the frequency boost time. Therefore, by adjusting the coupling strength, the value, and the time of the laser frequency boost, complete population transfer can be achieved. It has also shown that the numerical results are in agreement with the analytical calculations.

\section{REFERENCES}

[1] Y.-X. Du, X.-X. Yue, Z.-T. Liang, J.-Z. Li, H. Yan, and S.-L. Zhu, "Geometric atom interferometry with shortcuts to adiabaticity," Phys. Rev. A, Vol. 95, pp. 043608 (1-6), 2017.

[2] V.I. Yudin, A.V. Taichenachev, M.Y. Basalaev, and D.V. Kovalenko, "Dynamic regime of coherent population trapping and optimization of frequency modulation parameters in atomic clocks," Opt. Express, Vol. 25, pp. 2742 (1-10), 2017.

[3] T. Theis, Y. Feng, T. Wu, and W.S. Warren, "Composite and shaped pulses for efficient and robust pumping of disconnected eigenstates in magnetic resonance," J. Chem. Phys. Vol. 140, pp. 014201 (1-7), 2014.

[4] M. Saffman, T.G. Walker, and K. Mølmer, "Quantum information with Rydberg atoms," Rev. Mod. Phys. Vol. 82, pp. 2313-2363, 2010.

[5] F. Ahmadinouri, M. Hosseini, and F. Sarreshtedari, "Investigation of population transfer by sweeping the laser field in a finite time interval," Optik, Vol. 181, pp. 404-407, 2019.

[6] G. P. Djotyan, J. S. Bakos, Zs. Sörlei, and J. Szigeti, "Coherent control of atomic quantum states by single frequency-chirped laser pulses," Phys. Rev. Vol. 70, pp. 063406 (1-7), 2004.

[7] F. Sarreshtedari and M. Hosseini, "Tunable Landau-Zener transitions using continuousand chirped-pulse-laser couplings," Phys. Rev. A, Vol. 95, pp. 033834 (1-6), 2017.

[8] F. Ahmadinouri, M. Hosseini, and F. Sarreshtedari, "Investigation of robust population transfer using quadratically chirped laser interacting with a two-level system," Phys. Scr. Vol. 94, pp. 105404 (1-18), 2019. 
[9] F. Ahmadinouri, M. Hosseini, and F. Sarreshtedari, "Robust population transfer in a two-level system using finite chirping method," Phys. Scr. Vol. 94, pp. 085404 (113), 2019.

[10]F. Ahmadinouri, M. Hosseini, and F. Sarreshtedari, "Stimulated Raman adiabatic passage: effect of system parameters on population transfer," ArXiv 190200884 (122), 2019.

[11]N.V. Vitanov, A.A. Rangelov, B.W. Shore, and K. Bergmann, "Stimulated Raman adiabatic passage in physics, chemistry, and beyond," Rev. Mod. Phys. Vol. 89, pp. 015006 (1-78), 2017.

[12]M. Hosseini and F. Sarreshtedari, "Investigation of the laser controlled LandauZener mechanism in a coupled quantum system," J. Opt. Soc. Am. B Vol. 34, pp. 2097-2103, 2017.

[13] M. Wubs, K. Saito, S. Kohler, Y. Kayanuma, and P. Hanggi, "Landau-Zener transitions in qubits controlled by electromagnetic fields," New J. Phys. Vol. 7, pp. 218-218, 2005.

[14]H.T. Dung, R. Tanaś, and A.S. Shumovsky, "Collapses, revivals, and phase properties of the field in Jaynes-Cummings type models," Opt. Commun. Vol. 79, pp. 462-468, 1990.

[15]R.R. Schlicher, "Jaynes-Cummings model with atomic motion,"Opt. Commun. Vol. 70, pp. 97-102, 1989.

[16]B.W. Shore and P.L. Knight, "The jaynescummings model," J. Mod. Opt. Vol. 40, pp. 1195 (1-4), 1993.

[17]F. Bloch, "Nuclear induction," Phys. Rev. Vol. 70, pp. 460-474, 1946.

[18]I.I. Rabi, "Space quantization in a gyrating magnetic field," Phys. Rev. Vol. 51, pp. 652655, 1937.

[19]E.T. Jaynes and F.W. Cummings, "Comparison of quantum and semiclassical radiation theories with application to the beam maser," Proc. IEEE, Vol. 51, pp. 89-109, 1963.

[20]H. Walther, B.T.H. Varcoe, B.-G. Englert, and T. Becker, "Cavity quantum electrodynamics," Rep. Prog. Phys. Vol. 69, pp. 1325 (1-59), 2006.
[21]C.-G. Liao, C. Hu, Z.-K. Su, and S.-W. Chen, "The Collapse and Revival of Bell-nonlocaliy for Continuous Variables," Int. J. Theor. Phys. Vol. 54, pp. 1408-1416, 2015.

[22] R. Pakniat, M.K. Tavassoly, and M.H. Zandi, "Dynamics of Information Entropies of AtomField Entangled States Generated via the Jaynes-Cummings Model,"Commun. Theor. Phys. Vol. 65, pp. 266-272, 2016.

[23]P. Meystre and M.S. Zubairy, "Squeezed states in the Jaynes-Cummings model," Phys. Lett. A Vol. 89, pp. 390-392, 1982.

[24] J. Wang, H. Fang, and X. Xu, "Two-photon Jaynes-Cummings model interacting with the squeezed vacuum state solved by dressed-state method," Optik, Vol. 169, pp. 180-189, 2018.

[25] H.-M. Zou and M.-F. Fang, "Squeezing of light field in a dissipative Jaynes-Cummings model," J. Mod. Opt. Vol. 63, pp. 2279-2284, 2016

[26]A. Abragam, The Principles of Nuclear Magnetism, Oxford university press, 1961.

[27] A. Capua, C. Rettne r, S.-H. Yang, T. Phung, and S. S. P. Parkin, "Ensemble-averaged Rabi oscillations in a ferromagnetic $\mathrm{CoFeB}$ film," Nat. Commun. Vol. 8, pp. 16004 (1-7), 2017.

[28] T. Suzuki, R. Singh, M. Bayer, A. Ludwig, A. D. Wieck, and S. T. Cundiff, "Detuning dependence of Rabi oscillations in an InAs self-assembled quantum dot ensemble," Phys. Rev. B Vol. 97, pp. 161301 (1-7), 2018.

[29] Y.-Z. Xue, Z.-S. Chen, H.-Q. Ni, Z.-C. Niu, D.-S. Jiang, X.-M. Dou, and B.-Q. Sun, Chin. "Resonantly driven exciton Rabi oscillation in single quantum dots emitting at $1300 \mathrm{~nm}$," Phys. B Vol. 26, pp. 084202 (1-4), 2017.

[30]D. Leibfried, R. Blatt, C. Monroe, and D. Wineland, "Quantum dynamics of single trapped ions," Rev. Mod. Phys. Vol. 75, pp. 281-324, 2003.

[31]R. Puebla, J. Casanova, and M.B. Plenio, "A robust scheme for the implementation of the quantum Rabi model in trapped ions," New J. Phys. Vol. 18, pp. 113039 (1-21), 2016.

[32] K.R. Patton and U.R. Fischer, "Hybrid of superconducting quantum interference device and atomic Bose-Einstein condensate: An architecture for quantum information processing," Phys. Rev. A, Vol. 87, pp. 052303 (1-9), 2013. 
[33]F. Rossi and T. Kuhn, "Theory of ultrafast phenomena in photoexcited semiconductors," Rev. Mod. Phys. Vol. 74, pp. 895-950, 2002.

[34]P. Törmä and W.L. Barnes, "Strong coupling between surface plasmon polaritons and emitters: a review," Rep. Prog. Phys. Vol. 78, pp. 013901 (1-35), 2015.

[35] Y.A. Kosevich and J. Micromechanics "Nonlinear mechanical analogue of quantum Rabi oscillations in coherent output coupler for atoms in Bose-Einstein condensate," Mol. Phys. Vol. 01, pp. 1650007, 2016.

[36]D.A. Golter and H. Wang, "Optically driven Rabi oscillations and adiabatic passage of single electron spins in diamond," Phys. Rev. Lett. Vol. 112, pp. 116403-. 116407, 2014.

[37] J. M. Martinis, S. Nam, J. Aumentado, and C. Urbina, "Rabi oscillations in a large Josephson-junction qubit," Phys. Rev. Lett. Vol. 89, pp. 117901 (1-4), 2002.

[38]D. Suter, The physics of laser-atom interactions, Cambridge University Press, Cambridge, 1997.

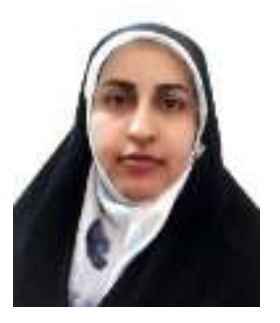

Fatemeh Ahmadinouri was born in Iran, in 1991. She received her B.Sc. degree from Arak University, Iran, in 2013 and M.Sc. degree in atomic and molecular physics from Shiraz University of Technology, Iran, in 2018. Her dissertation was concerned with the investigation of population transfer by applying chirped laser. She is a Ph.D. student of Laser Physics at Amirkabir University of Technology, Tehran, Iran. Her current research interests include random laser in biology, optical properties of fluorophores and chemodrugs, laser spectroscopy (LIF, LIBS) in medicine and laser- tissue interactions.

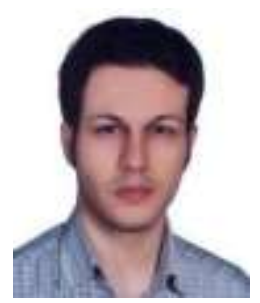

Mehdi Hosseini was born in Iran in 1983. He received the B.Sc., M.Sc., and Ph.D. degrees in physics from Sharif University of Technology, Tehran, Iran, in 2005, 2007, and 2011, respectively. After the Ph.D., he joined the Superconductive Electronics Research Laboratory (SERL) of Electrical Engineering Department of the Sharif University of Technology. He conducted research in the development of the superconductor thin film fabrication for two years as a postdoctoral fellow. He is currently a faculty member of the Physics Department of the Shiraz University of Technology. His research interests include statistical physics, solid-state physics, and superconductivity.

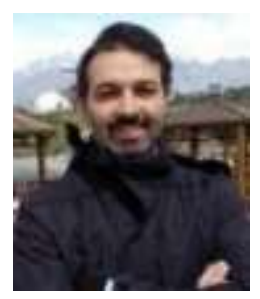

Farrokh Sarreshtedari was born in Iran in 1982. He received the B.Sc. M.Sc. and Ph.D. degrees from Sharif University of Technology, Tehran, Iran, in 2004, 2006, and 2012, respectively, all in electrical engineering. $\mathrm{He}$ is currently a faculty member in Department of Physics, University of Tehran. His research interests include magnetic resonance physics, Laser applications and optoelectronics. 
THIS PAGE IS INTENTIONALLY LEFT BLANK. 